\title{
LOCAL NULL CONTROLLABILITY OF A 1D STEFAN PROBLEM
}

\author{
E. FERNÁNDEZ-CARA, F. HERNÁNDEZ, J . LÍMACO
}

\begin{abstract}
The purpose of this article is to give a new proof of a null controllability result for a 1D free-boundary problem of the Stefan kind for a heat PDE. We introduce a method based on local inversion that, in contrast with other previous arguments, does not rely on any compactness property and can be generalized to higher dimensions.
\end{abstract}

\section{INTRODUCTION}

1.1. Motivation. Let $u_{0}:\left[0, L_{0}\right] \mapsto \mathbb{R}$, the open interval $\omega_{0} \Subset\left(0, L_{0}\right)$ and $T>0$ be given. The main goal in this paper is to find a control $v=v(x, t)$ such that the solution $(u, \ell)$ to the Stefan problem

$$
\left\{\begin{array}{lll}
u_{t}-u_{x x}=v \mathbb{1}_{\mathcal{O}} & \text { in } \quad Q_{\ell}, \\
u(0, t)=u(\ell(t), t)=0 & \text { for } t \in(0, T), \\
u(x, 0)=u_{0}(x) & \text { for } \quad x \in\left(0, L_{0}\right), \\
\ell^{\prime}(t)=-\frac{1}{k} u_{x}(\ell(t), t) & \text { for } t \in(0, T), & \text { (Stefan condition) } \\
\ell(0)=L_{0} & &
\end{array}\right.
$$

satisfies

$$
u(x, T)=0, \quad x \in(0, \ell(T)) .
$$

Here and in the sequel, $Q_{\ell}$ and $\mathcal{O}$ respectively denote

$$
Q_{\ell}=\{(x, t): 0<x<\ell(t), 0<t<T\} \text { and } \mathcal{O}=\omega_{0} \times(0, T)
$$

and $\mathbb{1}_{\mathcal{O}}$ is the characteristic function of $\mathcal{O}$.

Although this problem has already been considered in [12], it will be re-visited in this paper. In [12], problem (1.1) is reformulated and solved by introducing a suitable space $\mathcal{L}$ of functions $\ell:[0, T] \mapsto \mathbb{R}$ and a fixed-point mapping $\Lambda: \mathcal{L} \mapsto \mathcal{L}$ as follows:

$$
\Lambda(\ell)=L_{0}-\frac{1}{k} \int_{0}^{\cdot} u_{x}^{\ell}(\ell(s), s) d s,
$$

where $\left(u^{\ell}, v^{\ell}\right)$ is a well-chosen minimal energy solution to the null control problem:

$$
\begin{cases}u_{t}(x, t)-u_{x x}(x, t)=v(x, t) \mathbb{1}_{\mathcal{O}} & \text { in } \quad Q_{\ell}, \\ u(0, t)=u(\ell(t), t)=0 & \text { for } t \in(0, T), \\ u(x, 0)=u_{0}(x) & \text { for } x \in\left(0, L_{0}\right), \\ u(x, T)=0 & \text { for } x \in(0, \ell(T)) .\end{cases}
$$

The strategy to choose $\left(u^{\ell}, v^{\ell}\right)$ relies on the Fursikov-Imanuvilov formulation of the null controllability problem for the heat equation, see [18]. The authors prove that, for any small $u_{0}$, $\Lambda$ possesses at least one fixed point in $\mathcal{L}$, say $\ell^{\star}$. To this end, they check that the assumptions of Schauder's Fixed-Point Theorem are satisfied. As a by-product, a solution $\left(\ell^{\star}, u^{\ell^{\star}}, v^{\ell^{\star}}\right)$ to $(1.1)-(1.2)$ is proved to exist.

Unfortunately, it seems difficult to apply these ideas in a higher dimensional setting. Indeed, it is not clear at all how can we give a similar definition of $\Lambda$ and get, again, a continuous compact mapping (note that $\Lambda$ is compact in the one-dimensional case, among other reasons, because the functions in $\mathcal{L}$ can be viewed as parametrizations of curves, not surfaces).

For these reasons, with an extension in mind to other more interesting and realistic problems, we introduce in this paper another strategy that, instead of compactness, relies on local inversion, that is, completeness and contractivity. More precisely, we will use Liusternik's Inverse Function Theorem in Banach spaces.

An interesting by-product of the method used in this paper is that it leads in a natural way to a convergent iterative algorithm for the computation of null controls. This will be explained with more detail below, at the end of Section 4.

2000 Mathematics Subject Classification. 93B05, 93C20, 35K20, 35R35.

Key words and phrases. null controllability, free-boundary problems, 1D heat equation, Carleman estimates. 
The controllability of linear and nonlinear PDEs has been the objective of many papers the last decades. In the context of the linear and semilinear heat PDEs, the main contributions have been obtained in $[10,23,7,18,13,6]$. For parabolic free-boundary problems, controllability questions have been considered only in a few papers; see however $[3,20,12]$.

On the other hand, the solution of problems of the kind (1.1)-(1.2) is connected to several interesting applications. Among others, we can mention solidification processes (see [14, 15]) and also the following:

- The behavior of free surface flows, see [21, 28, 31].

- Fluid-solid interaction and related problems, see [5, 24, 30].

- Tumor growth modelling and other problems from mathematical biology, see [16, 17], etc.

In the sequel, $C$ denotes a generic positive constant; $C_{0}, C_{1}$, etc. are other positive (specific) constants; when it makes sense, the extension by zero in space of any function $f$ is denoted by $f^{*}$.

The main result in this paper is as follows:

Theorem 1.1. The free-boundary system (1.1)-(1.2) is locally null-controllable. More precisely, given a constant $L_{\star}$ such that $\sup \omega_{0}<L_{\star}<L_{0}$, there exists $\epsilon>0$ such that, if $u_{0} \in H_{0}^{1}\left(0, L_{0}\right)$ and $\left\|u_{0}\right\|_{H_{0}^{1}\left(0, L_{0}\right)}<\epsilon$, then there exist $\ell, v$ and $u$, with

$$
\ell \in C^{1}([0, T]), \quad L_{\star}<\inf _{t \in[0, T]} \ell(t), \quad v \in L^{2}(\mathcal{O}), \quad u \circ \Phi_{\ell}^{-1} \in C^{0}\left([0, T] ; H_{0}^{1}\left(0, L_{0}\right)\right),{ }^{1}
$$

that satisfy (1.1) and (1.2).

For the proof, we will first use a suitable change of variables that transforms (1.4) in a parabolic problem in a fixed cylindrical domain. Then, we will introduce two appropriate Banach spaces $\mathcal{X}, \mathcal{Y}$ and a mapping $\mathcal{F}: D \subset \mathcal{X} \mapsto \mathcal{Y}$ (defined on an appropriate subset $D$ ), and we will rewrite (1.1)-(1.2) as an equation of the form

$$
F(z, \ell, v)=\left(0, u_{0}\right), \quad(z, \ell, v) \in D \subset \mathcal{X} .
$$

Finally, we will check that the assumptions of Liusternik's Theorem are satisfied by $\mathcal{F}: D \subset$ $\mathcal{X} \mapsto \mathcal{Y}$ and, consequently, for any small $u_{0},(1.1)-(1.2)$ is solvable.

This paper is organized as follows. In Section 2, we give the details of the announced change of variables and we recall some previous results. In Section 3, we consider and solve a constrained null controllability problem for a linear parabolic system; this will be needed later to prove that the hypotheses of Liusternik's Theorem are fulfilled. Section 4 deals with the proof of Theorem 1.1. In Section 5, we present some additional comments and extensions. Finally, Section 6 is devoted to recall some technical results.

\section{Some PRevious Results}

As already mentioned, we will use a suitable change of variables in order to transform the non-cylindrical problem (1.4) into a cylindrical one.

2.1. Reduction to a fixed cylindrical domain. Let $\ell \in C^{1}([0, T])$ be given. We will construct a diffeomorphism $\Phi_{\ell}$ that maps the set $Q_{\ell}$ onto $Q_{L_{0}}=\left(0, L_{0}\right) \times(0, T)$ and coincides with the identity on the square $\left[0, L_{\star}\right] \times[0, T]$ for a fixed $L_{\star}$ such that $\sup \omega_{0}<L_{\star}<L_{0}$; see the figure below.

Construction of $\Phi_{\ell}$ : Fix a small $\delta>0$. Given $y>L_{\star}+2 \delta$, define $g(\cdot, y)$ as the linear interpolation of the points $\left(L_{\star}-\delta, L_{\star}-\delta\right),\left(L_{\star}+\delta, L_{\star}+\delta\right),\left(y-\delta, L_{0}-\delta\right),\left(y+\delta, L_{0}+\delta\right)$. Consider $g(\cdot, y)$ as defined on the whole line by extending the end segments toward infinity.

Specifically,

$$
g(x, y)= \begin{cases}x, & \text { if } x \leq L_{*}+\delta \\ L_{*}+\delta+\frac{\left(L_{*}-L_{0}+2 \delta\right)\left(x-L_{*}-\delta\right)}{\left(L_{*}+2 \delta-y\right)}, & \text { if } L_{*}+\delta<x<y-\delta \\ x-y+L_{0}, & \text { if } x>y-\delta\end{cases}
$$

\footnotetext{
${ }^{1} \Phi_{\ell}$ is a diffeomorphism constructed in subsection 2.1 for any $\ell \in C^{1}([0, T])$.
} 

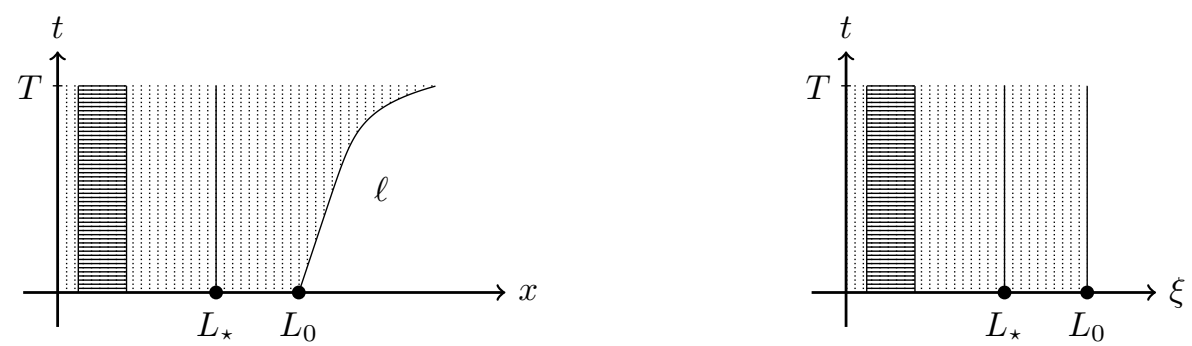

(A) Dotted region $Q_{\ell}$ and dashed region $\mathcal{O} \subset Q_{\ell}$. (в) Dotted region $Q_{L_{0}}$ and dashed region $\mathcal{O} \subset Q_{L_{0}}$.

On the other hand, let $\varphi$ be a nonnegative even smooth function with compact support contained in $(-\delta, \delta)$, which integral on this interval is equal to one: $\varphi \in C^{\infty}(\mathbb{R}), \varphi(x)=$ $\varphi(-x)$, supp $\varphi \subset(-\delta, \delta)$ and $\int_{\mathbb{R}} \varphi(x) d x=1$.

Let us define

$$
H(x, y)=(\varphi * g(\cdot, y))(x)
$$

It can be seen that $H$ is a smooth function on $\mathbb{R} \times\left(L_{\star}+2 \delta, \infty\right)$. Note that $H(y, y)=L_{0}$ and $H_{x}(y, y)=1$ for every $y>L_{\star}+2 \delta$. Moreover,

$$
\nabla H(x, y)=\left(\left(\varphi^{\prime} * g(\cdot, y)\right)(x),\left(\varphi * \partial_{y} g(\cdot, y)\right)(x)\right)
$$

where

$$
\partial_{y} g(x, y)= \begin{cases}0, & \text { if } x \leq L_{*}+\delta \\ \frac{\left(L_{*}-L_{0}+2 \delta\right)\left(x-L_{*}-\delta\right)}{\left(L_{*}+2 \delta-y\right)^{2}}, & \text { if } L_{*}+\delta<x<y-\delta \\ -1, & \text { if } x>y-\delta\end{cases}
$$

(see the figure below).

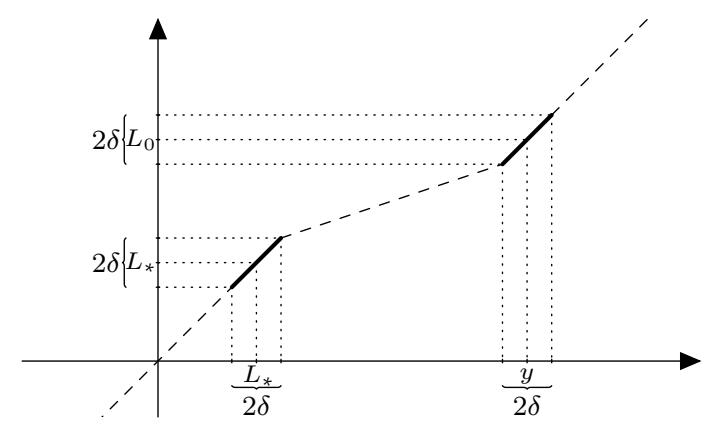

(A) Typical plot of $g(\cdot, y)$.

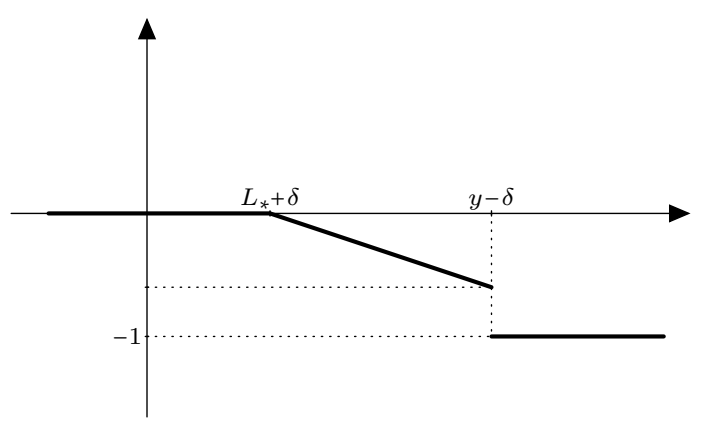

(B) Typical plot of $\partial_{y} g(\cdot, y)$.

The desired diffeomorphism is given as follows:

$$
\Phi_{\ell}: Q_{\ell} \mapsto Q_{L_{0}}, \quad \Phi_{\ell}(x, t)=(H(x, \ell(t)), t) .
$$

The change of variables $(\xi, t)=\Phi_{\ell}(x, t)$ transforms (1.4) into

$$
\begin{cases}M^{\ell} z=\tilde{v} \mathbb{1}_{\mathcal{O}}, & \text { in } \quad Q_{L_{0}} \\ z(0, t)=z\left(L_{0}, t\right)=0, & \text { for } t \in(0, T) \\ z(\xi, 0)=u_{0}(\xi), & \text { for } \xi \in\left(0, L_{0}\right) \\ z(\xi, T)=0, & \text { for } \xi \in\left(0, L_{0}\right)\end{cases}
$$

where

$$
\begin{gathered}
M^{\ell} z=z_{t}+a^{\ell} z_{\xi}-b^{\ell} z_{\xi \xi}, \\
z=u \circ \Phi_{\ell}^{-1}, \tilde{v}=v \circ \Phi_{\ell}^{-1} \text { and } \\
a^{\ell}(\xi, t)=H_{y}\left(H^{-1}(\xi, \ell(t)), \ell(t)\right) \ell^{\prime}(t)-H_{x x}\left(H^{-1}(\xi, \ell(t)), \ell(t)\right), \\
b^{\ell}(\xi, t)=H_{x}^{2}\left(H^{-1}(\xi, \ell(t)), \ell(t)\right) .
\end{gathered}
$$

Here, $H^{-1}$ denotes the inverse of $H$ with respect to the first coordinate (i.e. $H^{-1}(\xi, y):=(\varphi$ * $\left.g(\cdot, y))^{-1}(\xi)\right)$.

Observe that $a^{\ell} \in C(\bar{Q})$ and $b^{\ell} \in C^{1}(\bar{Q})$ whenever $\ell \in C^{1}[0, T]$.

Remark 2.1. Note that a slight modification in the construction of $\Phi$ actually permits to consider also controls acting on noncilindrical domains $\mathcal{O}$. 
2.2. Liusternik's Inverse Function Theorem. Let us consider the mapping

$$
\mathcal{F}(z, v, \ell)=\left(M^{\ell+L_{0}} z-v \mathbb{1}_{\mathcal{O}}, z(0)\right) .
$$

Note that $\mathcal{F}(0,0,0)=(0,0)$.

Later, our approach will be to locally invert this mapping, with the aim to ensure, for any small initial data $u_{0}$, the existence of a triplet $(z, v, \ell)$ such that $\mathcal{F}(z, v, \ell)=\left(0, u_{0}\right)$.

To accomplish this goal, we will apply the following local inversion result in Banach spaces, which is a consequence of the so-called Liusternik-Graves Theorem (see for instance [1]):

Theorem 2.2. Let $\mathcal{X}$ and $\mathcal{Y}$ be Banach spaces, let $D \subset \mathcal{X}$ be a non-empty open set and let $\mathcal{F}: D \subset \mathcal{X} \mapsto \mathcal{Y}$ be a $C^{1}$ mapping. Assume that $(\bar{x}, \bar{y}) \in D \times \mathcal{Y}$ is such that $\mathcal{F}(\bar{x})=\bar{y}$ and $\mathcal{F}^{\prime}(\bar{x}): \mathcal{X} \mapsto \mathcal{Y}$ is onto. Then, there exists $\delta>0$ such that, for every $y \in \mathcal{Y}$ satisfying $\|y-\bar{y}\|_{\mathcal{Y}}<\delta$, there exists at least one solution $x \in \mathcal{X}$ to the equation

$$
\mathcal{F}(x)=y, \quad x \in \mathcal{X} .
$$

Once the inversion is performed, we take $(z, v, \ell)=\mathcal{F}^{-1}\left(0, u_{0}\right)$ and, returning to the original coordinates, we see that the function $u(x, t):=z \circ \Phi_{\ell+L_{0}}(x, t)$ satisfies (1.1). As a matter of fact, what we can say by now is that the first and third lines of (1.1) are satisfied, but what about the other ones?

A major issue will be to find suitable Banach spaces $\mathcal{X}$ and $\mathcal{Y}$ such that, first, the hypotheses of Theorem 2.2 are fulfilled around $(0,0,0)$ and, second, $(z, v, \ell) \in \mathcal{X}$ implies that $u$ satisfies the remaining lines of (1.1), together with the zero final condition (1.2).

This will be achieved by imposing to the functions in $\mathcal{X}$ to satisfy the final null and boundary conditions together with the Stefan condition. Then, $\mathcal{Y}$ will be chosen such that $\mathcal{F}$ is well defined and $\mathcal{F}^{\prime}(0,0,0)$ is surjective. To get this last property, we need to solve a null controllability problem for a linear parabolic equation with and additional integral constraint, which requires the use of an improved Carleman's inequality, proved in Proposition 3.2.

\section{Constrained null controllability of linear parabolic equations}

Let us fix $u_{0} \in H_{0}^{1}\left(0, L_{0}\right)$ and $h \in L^{2}\left(\rho_{0}^{2} ; Q\right)$. The goal of this section is to solve the linear null controllability problem

$$
\left\{\begin{array}{lll}
M^{L_{0}} z(\xi, t)=v(\xi, t) \mathbb{1}_{\mathcal{O}}+h(\xi, t) & \text { in } \quad Q_{L_{0}}, \\
z(0, t)=z\left(L_{0}, t\right)=0 & \text { for } t \in(0, T), \\
z(\xi, 0)=u_{0}(\xi) & \text { for } \xi \in\left(0, L_{0}\right), \\
z(\xi, T)=0 & \text { for } \xi \in\left(0, L_{0}\right) .
\end{array}\right.
$$

with the additional constraint ${ }^{2}$

$$
\int_{0}^{T} z_{\xi}\left(L_{0}, s\right) d s=0 .
$$

Here $M^{L_{0}}$ denotes the operator defined by $(2.2)$ when $\ell(t) \equiv L_{0}$. That is

$$
M^{L_{0}}=\partial_{t}+a^{L_{0}} \partial_{\xi}-b^{L_{0}} \partial_{\xi \xi}
$$

It is not difficult to see (from (2.3), (2.4) and the definition of $H$ ) that $a^{L_{0}}=0$ and $b^{L_{0}}=1$. Nevertheless, we maintain the notation given above because most calculations performed below remain valid if we replace the constant $L_{0}$ for a fixed function $\ell_{0}$ such that $\ell_{0}(0)=L_{0}$.

The main tool will be a modified (or improved) Carleman's estimate that will be established for the solutions to an adjoint problem. Ideas of this kind seem to have been used for the first time in [27] and has led since then to the solution of several constrained control problems; see for example $[26,25]$.

Let us first introduce some notation. Thus, let us fix a nonnegative function $\alpha_{0} \in C^{2}\left(\left[0, L_{0}\right]\right)$ such that $\alpha_{0}(0)=\alpha_{0}\left(L_{0}\right)=0$ and $\left|\alpha_{0}^{\prime}(x)\right|>0$ for all $x \in\left[0, L_{0}\right] \backslash \omega_{0}$. Given $\lambda>0$, let us set

$$
\phi(x, t):=\frac{e^{\lambda \alpha_{0}(x)}}{m(t)}, \quad \tilde{\alpha}(x, t):=e^{2 \lambda\left\|\alpha_{0}\right\|_{\infty}}-e^{\lambda \alpha_{0}(x)} \quad \text { and } \quad \alpha(x, t):=\frac{\tilde{\alpha}(x)}{m(t)},
$$

where $m \in C^{\infty}([0, T])$ is such that

$$
m(t)= \begin{cases}T^{2} / 4 & \text { for } t \in[0, T / 2] \\ t(T-t) & \text { for } t \in[T / 2, T]\end{cases}
$$

Then the following well known global Carleman estimate holds (see [18]):

\footnotetext{
${ }^{2} \mathrm{~A}$ justification for the introduction of this constraint is given in Section 4 after the proof of Lemma 4.3.
} 
Proposition 3.1 (Carleman inequality). There exist positive constants $\tilde{\lambda}, \tilde{s}$ and $C$ (depending on $L_{\star}, \omega_{0}$ and $\left.T\right)$ such that, for any $s \geq \tilde{s}$ and $\lambda \geq \tilde{\lambda}$,

$$
\begin{array}{r}
\iint_{Q_{L_{0}}} e^{-2 s \alpha}\left[(s \phi)^{-1}\left(\left|p_{t}\right|^{2}+\left|p_{\xi \xi}\right|^{2}\right)+\lambda^{2}(s \phi)\left|p_{\xi}\right|^{2}+\lambda^{4}(s \phi)^{3}|p|^{2}\right] d \xi d t \\
\leq C\left(\iint_{Q_{L_{0}}} e^{-2 s \alpha}\left|\left(M^{L_{0}}\right)^{*}(p)\right|^{2} d \xi d t+\iint_{\mathcal{O}} e^{-2 s \alpha} \lambda^{4}(s \phi)^{3}|p|^{2} d \xi d t\right) .
\end{array}
$$

for any $p \in H^{2,1}\left(Q_{L_{0}}\right)$ such that $p(0, t)=p\left(L_{0}, t\right)=0$ for $0 \leq t \leq T$.

In general, $\left(M^{\ell_{0}}\right)^{*}(p):=-p_{t}-\left(a^{\ell_{0}} p\right)_{\xi}-\left(b^{\ell_{0}} p\right)_{\xi \xi}$ corresponds to the formal adjoint of $M^{\ell_{0}}$. The preceding result remains in force for such operators because of the regularity of $a_{0}^{\ell}$ and $b^{\ell_{0}}$ mentioned at the end of Subsection 2.1. In our current setting we have $\left(M^{L_{0}}\right)^{*}(p):=-p_{t}-p_{\xi \xi}$.

From here on we use the following notation for the weight functions in (3.4):

$$
\rho=e^{s \alpha}, \quad \rho_{0}=\lambda^{-2} e^{s \alpha}(s \phi)^{-3 / 2}, \quad \rho_{1}=\lambda^{-1} e^{s \alpha}(s \phi)^{-1 / 2}, \quad \rho_{2}=e^{s \alpha}(s \phi)^{1 / 2} .
$$

In order to solve the constrained problem (3.1)-(3.2), we will first ignore the integral restriction. Thus, from the results in [18], we know that there exists $(\bar{z}, \bar{v})$ in $L^{2}\left(\rho^{2} ; Q_{L_{0}}\right) \times L^{2}\left(\rho_{0}^{2} ; \mathcal{O}\right)$ (that will be fixed from now on and up to the end of this section), such that

$$
\begin{cases}M^{L_{0}} \bar{z}=\bar{v} \mathbb{1}_{\mathcal{O}}+h, & \text { in } \quad Q_{L_{0}}, \\ \bar{z}(0, t)=\bar{z}\left(L_{0}, t\right)=0, & \text { for } t \in(0, T), \\ \bar{z}(\xi, 0)=u_{0}(\xi), & \text { for } \xi \in\left(0, L_{0}\right),\end{cases}
$$

and

$$
\|\bar{z}\|_{L^{2}\left(\rho^{2} ; Q_{L_{0}}\right)}^{2}+\|\bar{v}\|_{L^{2}\left(\rho_{0}^{2} ; \mathcal{O}\right)}^{2} \leq C\left(\iint_{Q_{0}} \rho_{0}^{2}|h|^{2} d \xi d t+\int_{0}^{L_{0}}\left|u_{0}\right|^{2} d \xi\right) .
$$

Moreover, it is not difficult to see that

$$
\bar{\eta}^{2}:=\left(\int_{0}^{T} \bar{z}_{\xi}\left(L_{0}, s\right) d s\right)^{2} \leq C\left(\iint_{Q_{0}} \rho_{0}^{2}|h|^{2} d \xi d t+\int_{0}^{L_{0}}\left|u_{0}\right|^{2} d \xi\right) .
$$

Let us denote by $Z(v)$ the solution (in $L^{2}\left(0, T ; H^{2}\left(0, L_{0}\right)\right)$ ) to the problem

$$
\begin{cases}M^{L_{0}} z=v \mathbb{1}_{\mathcal{O}} & \text { in } \quad Q_{L_{0},} \\ z(0, t)=z\left(L_{0}, t\right)=0 & \text { for } \quad t \in(0, T), \\ z(\xi, 0)=0 & \text { for } \quad \xi \in\left(0, L_{0}\right),\end{cases}
$$

and let us introduce the family of admissible controls

$$
\mathcal{V}_{\mathrm{ad}}=\left\{v \in L^{2}\left(\rho_{0}^{2} ; \mathcal{O}\right): \int_{0}^{T}(Z(v))_{\xi}\left(L_{0}, s\right) d s=\bar{\eta}\right\}
$$

In terms of the linear (nonlocal) differential operator

$$
N: L^{2}\left(\rho_{0}^{2} ; \mathcal{O}\right) \mapsto L^{2}(0, T), \quad N(v)=(Z(v))_{\xi}\left(L_{0}, s\right),
$$

one has

$$
\mathcal{V}_{\mathrm{ad}}=\left\{v \in L^{2}\left(\rho_{0}^{2} ; \mathcal{O}\right):\left\langle N^{*} 1, v\right\rangle_{L^{2}\left(\rho_{0}^{-2} ; \mathcal{O}\right) \times L^{2}\left(\rho_{0}^{2} ; \mathcal{O}\right)}=\bar{\eta}\right\},
$$

where $N^{*}: L^{2}(0, T) \mapsto L^{2}\left(\rho_{0}^{-2} ; \mathcal{O}\right)$ denotes the adjoint of $N$ and 1 is the unit constant function.

To simplify the notation, we write the previous duality product in the form $\langle\cdot, \cdot\rangle_{\rho_{0}^{-2} \times \rho_{0}^{2}}$. Note that $\left\langle w^{*}, v\right\rangle_{\rho_{0}^{-2} \times \rho_{0}^{2}}$ coincides with $\iint_{\mathcal{O}} w^{*} v d \xi d t$; in particular, we see that $\langle p, p\rangle_{\rho_{0}^{-2} \times \rho_{0}^{2}}$ is well defined for any $p \in C^{2}\left(\overline{Q_{0}}\right)$.

It is possible to give an explicit expression for the operator $N^{*}$. In fact, integrating the identity

$$
M^{L_{0}}(y) \varphi-y\left(M^{L_{0}}\right)^{*}(\varphi)=(y \varphi)_{t}+y \varphi_{\xi \xi}-y_{\xi \xi} \varphi
$$

on $Q_{L_{0}}$, where $y=Z(v)$ and $\varphi$ is the solution of

$$
\begin{cases}\left(M^{L_{0}}\right)^{*} \varphi=0 & \text { in } \quad Q_{L_{0}} \\ \varphi(0, t)=0 \quad \varphi\left(L_{0}, t\right)=h(t) & \text { for } t \in(0, T) \\ \varphi(\xi, 0)=0 & \text { for } \xi \in\left(0, L_{0}\right)\end{cases}
$$

we obtain

$$
\left.\iint_{Q_{L_{0}}} v \varphi\right|_{\mathcal{O}} d \xi d t=-\int_{0}^{T} Z(v)_{\xi}\left(L_{0}, t\right) h(t) d t .
$$

In other words, $N^{*}(h)=-\left.\varphi\right|_{\mathcal{O}}$ where $\varphi$ is the solution of (3.9). 
Now, let us introduce the space

$$
\mathcal{P}_{0}:=\left\{p \in C^{2}\left(\overline{Q_{0}}\right): p(0, t)=p\left(L_{0}, t\right)=0 \quad \forall t \in[0, T]\right\},
$$

endowed with the scalar product

$$
\begin{aligned}
\langle\langle p, \bar{p}\rangle= & \iint_{Q_{L_{0}}} \rho^{-2}\left(M^{L_{0}}\right)^{*}(p)\left(M^{L_{0}}\right)^{*}(\bar{p}) d \xi d t \\
& +\iint_{Q_{L_{0}}} \rho_{0}^{-2}\left(p-\widehat{P}\left(\left.\rho_{0}^{-2} p\right|_{\mathcal{O}}\right)\left(\bar{p}-\widehat{P}\left(\left.\rho_{0}^{-2} \bar{p}\right|_{\mathcal{O}}\right) \mathbb{1}_{\mathcal{O}} d \xi d t\right.\right.
\end{aligned}
$$

where $\widehat{P}(q)$ stands for

$$
\widehat{P}(q)=\frac{\left\langle N^{*} 1, q\right\rangle_{\rho_{0}^{-2} \times \rho_{0}^{2}}}{\left\|N^{*} 1\right\|_{L^{2}\left(\rho_{0}^{-2}\right)}^{2}} N^{*} 1 .
$$

Clearly, the first integral in (3.10) is finite for $p, \bar{p} \in P_{0}$. For the well definition of the second integral, it is enough to note that $p \in P_{0}$ implies that $\left.\rho_{0}^{-2} p\right|_{\mathcal{O}} \in L^{2}\left(\rho_{0}^{2} ; \mathcal{O}\right)$, therefore $\widehat{P}\left(\left.\rho_{0}^{-2} p\right|_{\mathcal{O}}\right)$ is well defined and belongs to $L^{2}\left(\rho_{0}^{-2} ; \mathcal{O}\right)$.

Let us check that $\langle\cdot \cdot \cdot \cdot\rangle$ is in fact a scalar product in $\mathcal{P}_{0}$. Suppose that $\langle\langle p, p\rangle\rangle=0$ for some $p \in \mathcal{P}_{0}$. Then

$$
\begin{cases}\left(M^{L_{0}}\right)^{*}(p)=0, & \text { in } \quad Q_{L_{0}} \\ p(0, t)=p\left(L_{0}, t\right)=0, & \text { for } t \in(0, T)\end{cases}
$$

and $p=\beta N^{*} 1$ in $\mathcal{O}$, where $\beta=\frac{\left\langle N^{*} 1,\left.\rho_{0}^{-2} p\right|_{\mathcal{O}}\right\rangle_{\rho_{0}^{-2} \times \rho_{0}^{2}}}{\left\|N^{*} 1\right\|_{L^{2}\left(\rho_{0}^{-2}\right)}}$.

It follows from the characterization of $N^{*}$ given above that $N^{*} 1=-\left.\tilde{\varphi}\right|_{\mathcal{O}}$, where $\tilde{\varphi}$ is the solution of (3.9) with $h(t) \equiv 1$. As a consequence,

$$
\left\{\begin{array}{lll}
\left(M^{L_{0}}\right)^{*}(p+\beta \tilde{\varphi})=0, & \text { in } Q_{L_{0}} \\
p+\beta \tilde{\varphi}=0, & \text { in } \mathcal{O} .
\end{array}\right.
$$

By unique continuation, which is a direct consequence of Carleman's inequality (3.4), we have $p=$ $-\beta \tilde{\varphi}$ in $Q_{L_{0}}$. Hence, in particular, $p\left(L_{0}, t\right)=-\beta \tilde{\varphi}\left(L_{0}, t\right)$, which implies $\beta=0$ and, consequently, $p=0$.

In the sequel, we will use a well known uniqueness-compactness result. It is the following:

Let $X$ and $Y$ be reflexive Banach spaces and assume that $A, B \in \mathcal{L}(X ; Y), A$ is injective and $B$ is compact. Also, assume that one has

$$
\|z\|_{X} \leq C_{1}\left(\|A z\|_{Y}+\|B z\|_{Y}\right) \quad \forall z \in X .
$$

Then, there exists $C_{2}>0$ such that

$$
\|z\|_{X} \leq C_{2}\|A z\|_{Y} \quad \forall z \in X .
$$

Indeed, if this were not the case, there would exist $z_{1}, z_{2}, \ldots$ in $X$ such that

$$
\left\|z_{n}\right\|_{X}=1 \text { and }\left\|A z_{n}\right\|_{Y}<\frac{1}{n} \quad \forall n \geq 1
$$

We can assume that, for some $z_{*} \in X, z_{n} \rightarrow z_{*}$ weakly in $X$ and $B z_{n} \rightarrow B z_{*}$ strongly in $Y$ as $n \rightarrow+\infty$. Since $A z_{*}=0$, we necessarily have $z_{*}=0$ and therefore $\left\|B z_{n}\right\|_{Y} \rightarrow 0$.

But we also have from (3.11) that

$$
1=\left\|z_{n}\right\|_{X} \leq C_{1}\left(\left\|A z_{n}\right\|_{Y}+\left\|B z_{n}\right\|_{Y}\right) \leq C_{1}\left(\frac{1}{n}+\left\|B z_{n}\right\|_{Y}\right) \quad \forall n \geq 1,
$$

whence we arrive to a contradiction.

In the sequel, we will denote by $P$ the completion of $P_{0}$ for the scalar product $\langle\langle\cdot, \cdot\rangle$ :

$$
\mathcal{P}:=\overline{\mathcal{P}_{0}}\langle\cdot \cdot \cdot\rangle
$$

Proposition 3.2 (Carleman inequality). There exist positive constants $\lambda_{0}, s_{0}$ and $C$ (depending on $L_{0}, \omega$ and $T$ ) such that, for any $s \geq s_{0}$ and $\lambda \geq \lambda_{0}$, one has:

$$
\begin{aligned}
& I(p)=\iint_{Q_{L_{0}}} e^{-2 s \alpha}\left[(s \phi)^{-1}\left(\left|p_{t}\right|^{2}+\left|p_{\xi \xi}\right|^{2}\right)+\lambda^{2}(s \phi)\left|p_{\xi}\right|^{2}+\lambda^{4}(s \phi)^{3}|p|^{2}\right] d \xi d t \\
\leq & C\left(\iint_{Q_{L_{0}}} e^{-2 s \alpha}\left|\left(M^{L_{0}}\right)^{*}(p)\right|^{2} d \xi d t+\iint_{\mathcal{O}} e^{-2 s \alpha} \lambda^{4}(s \phi)^{3}\left|p-\widehat{P}\left(\left.\rho_{0}^{-2} p\right|_{\mathcal{O}}\right)\right|^{2} d \xi d t\right) .
\end{aligned}
$$

for any $p \in \mathcal{P}$. 
Proof. Recall that

$$
\widehat{P}(p)=\frac{\left\langle N^{*} 1, p\right\rangle_{\rho_{0}^{-2} \times \rho_{0}^{2}}}{\left\|N^{*} 1\right\|_{L^{2}\left(\rho_{0}^{-2}\right)}^{2}} N^{*} 1 \quad \forall p \in P .
$$

The image of $\widehat{P}$ is a one-dimensional vector space; thus, $\widehat{P}$ can be viewed as a compact operator.

On the other hand, in view of the usual Carleman's estimates (Proposition 3.1), we have

$$
\begin{aligned}
I(p) & \leq C\left(\iint_{Q_{L_{0}}} \rho^{-2}\left|\left(M^{L_{0}}\right)^{*}(p)\right|^{2} d \xi d t+\iint_{\mathcal{O}} \rho_{0}^{-2}|p-\widehat{P} p|^{2} d \xi d t\right. \\
& \left.+\iint_{\mathcal{O}} \rho_{0}^{-2}|\widehat{P} p|^{2} d \xi d t\right) .
\end{aligned}
$$

We can rewrite this in the form

$$
I(p) \leq C\left(\|A p\|_{L^{2}\left(Q_{L_{0}}\right) \times L^{2}(\mathcal{O})}^{2}+\|B p\|_{L^{2}\left(Q_{L_{0}}\right)}^{2}\right),
$$

where the linear operators $A$ and $B$, are respectively given by

$$
A p:=\left(\rho^{-1}\left(M^{L_{0}}\right)^{*}(p),\left.\rho_{0}^{-1}(p-\widehat{P} p)\right|_{\mathcal{O}}\right)
$$

and

for all $p \in \mathcal{P}_{0}$.

$$
B p:=\left.\rho_{0}^{-1} \widehat{P} p\right|_{\mathcal{O}}
$$

We thus see that $A$ and $B$ are respectively injective and compact. Hence, the uniquenesscompactness argument can be applied in this framework. This means that we can write an inequality similar to (3.13) where the last integral is skipped. In other words, there exists a new constant $C$ such that

$$
\begin{aligned}
I(p) & \leq C\left(\iint_{Q_{L_{0}}} \rho^{-2}\left|\left(M^{L_{0}}\right)^{*}(p)\right|^{2} d \xi d t+\iint_{\mathcal{O}} \rho_{0}^{-2}|p-\widehat{P} p|^{2} d \xi d t\right) . \\
& =C\langle\langle p, p\rangle
\end{aligned}
$$

for all $p \in \mathcal{P}$.

The main result of this section is the following:

Theorem 3.3. Assume that $u_{0} \in H_{0}^{1}\left(0, L_{0}\right)$ and $\rho_{0} h \in L^{2}\left(Q_{L_{0}}\right)$. Then, there exist $v \in L^{2}(\mathcal{O})$ and $z \in L^{2}\left([0, T] ; H_{0}^{1}\left(0, L_{0}\right) \cap H^{2}\left(0, L_{0}\right)\right)$ such that $z_{t} \in L^{2}\left([0, T] ; L^{2}\left(0, L_{0}\right)\right)$ satisfying (3.1) and (3.2). Furthermore, the following estimate holds:

$$
\|\rho z\|_{L^{2}\left(Q_{L_{0}}\right)}^{2}+\left\|\rho_{0} v\right\|_{L^{2}(\mathcal{O})}^{2} \leq C\left(\iint_{Q_{0}} \rho_{0}^{2}|h|^{2} d \xi d t+\int_{0}^{L_{0}}\left|u_{0}\right|^{2} d \xi\right)
$$

Proof. Let $\mathcal{L}$ be the linear form given by

$$
\mathcal{L}: \mathcal{P} \mapsto \mathbb{R}, \quad \mathcal{L}(q):=\iint_{\mathcal{O}} \bar{v} q d \xi d t
$$

Using Schwarz's inequality, the estimate (3.6) and the Carleman's estimate in Proposition (3.2), we get:

$$
\begin{aligned}
|\mathcal{L}(q)| & \leq\left(\iint_{Q_{0}} \rho_{0}^{2}|\bar{v}|^{2} d \xi d t\right)^{1 / 2}\left(\iint_{Q_{0}} \rho_{0}^{-2}|q|^{2} d \xi d t\right)^{1 / 2} \\
& \leq C\left(\iint_{Q_{0}} \rho_{0}^{2}|h|^{2} d \xi d t+\int_{0}^{L_{0}}\left|u_{0}\right|^{2} d \xi\right)^{1 / 2}\langle\langle q, q\rangle\rangle^{1 / 2} .
\end{aligned}
$$

Therefore, $\mathcal{L} \in \mathcal{P}^{\prime}$ and, from Lax-Milgram's Theorem, we deduce the existence of a unique $p$ satisfying

$$
\langle p, q\rangle\rangle=\mathcal{L}(q) \quad \forall q \in \mathcal{P}, \quad p \in \mathcal{P} .
$$

Then,

$$
\begin{gathered}
\iint_{Q}\left(\rho^{-2}\left(M^{L_{0}}\right)^{*}(p)\left(M^{L_{0}}\right)^{*}(q)+\rho_{0}^{-2}\left(p-\widehat{P}\left(\left.\rho_{0}^{-2} p\right|_{\mathcal{O}}\right)\right)\left(q-\widehat{P}\left(\left.\rho_{0}^{-2} q\right|_{\mathcal{O}}\right)\right) \mathbb{1}_{\mathcal{O}}\right) d \xi d t \\
=\iint_{\mathcal{O}} \bar{v} q d \xi d t \quad \forall q \in \mathcal{P} .
\end{gathered}
$$


Let us introduce $\tilde{z}:=\rho^{-2}\left(M^{L_{0}}\right)^{\star}(p)$ and $\tilde{v}:=\left(\bar{v}-\rho_{0}^{-2}\left(p-\widehat{P}\left(\left.\rho_{0}^{-2} p\right|_{\mathcal{O}}\right)\right)\right) \mathbb{1}_{\mathcal{O}}$. In particular, $\left\langle N^{*} 1, \tilde{v}\right\rangle_{\rho_{0}^{-2} \times \rho_{0}^{2}}=\bar{\eta}$, that is, $\tilde{v}$ belongs to $\mathcal{V}_{\text {ad }}$. Noting that

$$
\iint_{Q} \rho_{0}^{-2}\left(p-\widehat{P}\left(\left.\rho_{0}^{-2} p\right|_{\mathcal{O}}\right)\right) \widehat{P}\left(\left.\rho_{0}^{-2} q\right|_{\mathcal{O}}\right) \mathbb{1}_{\mathcal{O}} d \xi d t=0
$$

we see that

$$
\iint_{Q} \tilde{z}\left(M^{L_{0}}\right)^{*}(q) d \xi d t=\iint_{Q} q \tilde{v} \mathbb{1}_{\mathcal{O}} d \xi d t \quad \forall q \in \mathcal{P} .
$$

Consequently, $\tilde{z}$ is a solution by transposition of

$$
\left\{\begin{array}{lll}
M^{L_{0}} \tilde{z}(\xi, t)=\tilde{v}(\xi, t) \mathbb{1}_{\mathcal{O}} & \text { in } & Q_{L_{0}}, \\
\tilde{z}(0, t)=\tilde{z}\left(L_{0}, t\right)=0 & \text { for } & t \in(0, T), \\
\tilde{z}(\xi, 0)=0 & \text { for } & \xi \in\left(0, L_{0}\right) .
\end{array}\right.
$$

Actually, since $\tilde{v} \in L^{2}\left(\rho_{0}^{2} ; \mathcal{O}\right),(3.17)$ possesses a unique weak solution $\theta$ satisfying

$$
\theta \in L^{2}\left(0, T ; H^{2}\left(0, L_{0}\right)\right), \quad \theta_{t} \in L^{2}\left(0, T ; L^{2}\left(0, L_{0}\right)\right),
$$

which has to coincide with $\tilde{z}$.

As an additional consequence of (3.16), we also have that

$$
\|\rho \tilde{z}\|_{L^{2}\left(Q_{L_{0}}\right)}^{2}+\left\|\rho_{0}(\tilde{v}-\bar{v})\right\|_{L^{2}(\mathcal{O})}^{2} \leq C\left(\iint_{Q_{0}} \rho_{0}^{2}|h|^{2} d \xi d t+\int_{0}^{L_{0}}\left|u_{0}\right|^{2} d \xi\right) .
$$

The proof of Theorem 3.3 follows from (3.5)-(3.7), (3.17) and (3.18), by taking $z=\bar{z}-\tilde{z}$ and $v=\bar{v}-\tilde{v}$.

We will end this section by establishing some important estimates for the state-control pairs $(z, v)$ that satisfy $(3.1)$.

Lemma 3.4. Suppose that $(z, v) \in L^{2}\left(\rho^{2} ; Q_{L_{0}}\right) \times L^{2}\left(\rho_{0}^{2} ; \mathcal{O}\right)$ solves $(3.1)$ with $u_{0} \in H_{0}^{1}\left(0, L_{0}\right)$ and $h \in L^{2}\left(\rho_{0}^{2} ; Q_{L_{0}}\right)$. Then

$$
\begin{aligned}
\left\|\hat{\rho} z_{\xi}\right\|_{L^{2}\left(Q_{L_{0}}\right)}^{2} & \leq C\left(\left\|u_{0}\right\|_{L^{2}\left(0, L_{0}\right)}^{2}+\|h\|_{L^{2}\left(\rho_{0}^{2} ; Q_{L_{0}}\right)}^{2}+\|z\|_{L^{2}\left(\rho^{2} ; Q_{L_{0}}\right)}^{2}+\|v\|_{L^{2}\left(\rho_{0}^{2} ; \mathcal{O}\right)}^{2}\right), \\
\left\|\rho^{\star} z_{t}\right\|_{L^{2}\left(Q_{L_{0}}\right)}^{2} & \leq C\left(\left\|u_{0}\right\|_{H_{0}^{1}\left(0, L_{0}\right)}^{2}+\|h\|_{L^{2}\left(\rho_{0}^{2} ; Q_{L_{0}}\right)}^{2}+\|z\|_{L^{2}\left(\rho^{2} ; Q_{L_{0}}\right)}^{2}+\|v\|_{L^{2}\left(\rho_{0}^{2} ; \mathcal{O}\right)}^{2}\right), \\
\left\|\rho^{\star} z_{\xi \xi}\right\|_{L^{2}\left(Q_{L_{0}}\right)}^{2} & \leq C\left(\left\|u_{0}\right\|_{H_{0}^{1}\left(0, L_{0}\right)}^{2}+\|h\|_{L^{2}\left(\rho_{0}^{2} ; Q_{L_{0}}\right)}^{2}+\|z\|_{L^{2}\left(\rho^{2} ; Q_{L_{0}}\right)}^{2}+\|v\|_{L^{2}\left(\rho_{0}^{2} ; \mathcal{O}\right)}^{2}\right),
\end{aligned}
$$

where $\hat{\rho}=m \rho$ and $\rho^{\star}=m^{2} \rho$.

Proof. Let us prove each estimate separately.

- Proof of (3.19): Multiplying the first line of (3.1) by $\hat{\rho}^{2} z$, and integrating in space, we have

$$
\begin{aligned}
\frac{1}{2} \frac{d}{d t} \int_{0}^{L_{0}} \hat{\rho}^{2}|z|^{2} d \xi & +\int_{0}^{L_{0}} b^{\ell} \hat{\rho}^{2}\left|z_{\xi}\right|^{2} d \xi=\int_{0}^{L_{0}}\left(v \mathbb{1}_{\mathcal{O}}+h\right) \hat{\rho}^{2} z d \xi+\int_{0}^{L_{0}} \hat{\rho} \hat{\rho}_{t}|z|^{2} d \xi \\
& -2 \int_{0}^{L_{0}} b^{\ell} \hat{\rho} \hat{\rho}_{\xi} z z_{\xi} d \xi-\int_{0}^{L_{0}}\left(b_{\xi}^{\ell}+a^{\ell}\right) \hat{\rho}^{2} z z_{\xi} d \xi
\end{aligned}
$$

The following estimates are obtained by calculating explicitly $\hat{\rho}_{t}$ and $\hat{\rho}_{\xi}$, and then using Hölder and Young inequalities:

$$
\begin{gathered}
\int_{0}^{L_{0}}\left|\hat{\rho} \hat{\rho}_{t}\right||z|^{2} d \xi \leq C \int_{0}^{L_{0}} \rho^{2}|z|^{2} d \xi \\
\int_{0}^{L_{0}}\left|\hat{\rho} \hat{\rho}_{\xi} z z_{\xi}\right| d \xi \leq C \beta \int_{0}^{L_{0}} \rho^{2}\left|z_{\xi}\right|^{2} d \xi+\frac{C}{4 \beta} \int_{0}^{L_{0}} \rho^{2}|z|^{2} d \xi
\end{gathered}
$$

and

$$
\int_{0}^{L_{0}}\left|H \hat{\rho}^{2} z\right| d \xi \leq C \beta \int_{0}^{L_{0}} \rho_{0}^{2} H^{2} d \xi+\frac{C}{4 \beta} \int_{0}^{L_{0}} \rho^{2}|z|^{2} d \xi,
$$

where $\beta$ is any positive number and $H$ is any function.

Since $a^{\ell}, b^{\ell}$ and $b_{\xi}^{\ell}$ are bounded and $b^{\ell}$ is positive and bounded from below, we can choose $\beta$ in the previous estimates such that, after integrating in $(0, t)$ for $0<t<T$ and taking limits as $t$ goes to $T$, we arrive at (3.19). 
- Proof of (3.20): Multiplying the first line of (3.1) by $\left(\rho^{\star}\right)^{2} z_{t}$ and integrating in space, we have:

$$
\begin{aligned}
\int_{0}^{L_{0}}\left(\rho^{\star}\right)^{2}\left|z_{t}\right|^{2} d \xi & +\frac{1}{2} \frac{d}{d t} \int_{0}^{L_{0}} b^{\ell}\left(\rho^{\star}\right)^{2}\left|z_{\xi}\right|^{2} d \xi=\int_{0}^{L_{0}}\left(v \mathbb{1}_{\mathcal{O}}+h\right)\left(\rho^{\star}\right)^{2} z_{t} d \xi-2 \int_{0}^{L_{0}} \rho^{\star} \rho_{\xi}^{\star} z_{\xi} z_{t} d \xi \\
& +\int_{0}^{L_{0}} b^{\ell} \rho^{\star} \rho_{t}^{\star}\left|z_{\xi}\right|^{2} d \xi+\frac{1}{2} \int_{0}^{L_{0}} b_{t}^{\ell}\left(\rho^{\star}\right)^{2}\left|z_{\xi}\right|^{2} d \xi-\int_{0}^{L_{0}}\left(b_{\xi}^{\ell}+a^{\ell}\right)\left(\rho^{\star}\right)^{2} z_{t} z_{\xi} d \xi
\end{aligned}
$$

Now, we argue as in the previous paragraph. First, we get

$$
\begin{gathered}
\int_{0}^{L_{0}}\left|\rho^{\star} \rho_{t}^{\star}\right|\left|z_{\xi}\right|^{2} d \xi \leq C \int_{0}^{L_{0}} \hat{\rho}^{2}\left|z_{\xi}\right|^{2} d \xi, \quad \int_{0}^{L_{0}}\left(\rho^{\star}\right)^{2}\left|z_{\xi}\right|^{2} d \xi \leq C \int_{0}^{L_{0}} \hat{\rho}^{2}\left|z_{\xi}\right|^{2} d \xi \\
\int_{0}^{L_{0}}\left|\rho^{\star} \rho_{\xi}^{\star} z_{\xi} z_{t}\right| d \xi \leq C \beta \int_{0}^{L_{0}}\left(\rho^{\star}\right)^{2}\left|z_{t}\right|^{2} d \xi+\frac{C}{4 \beta} \int_{0}^{L_{0}} \hat{\rho}^{2}\left|z_{\xi}\right|^{2} d \xi
\end{gathered}
$$

and

$$
\int_{0}^{L_{0}}\left|\left(\rho^{\star}\right)^{2} z_{t} H\right| d \xi \leq C \beta \int_{0}^{L_{0}}\left(\rho^{\star}\right)^{2}\left|z_{t}\right|^{2} d \xi+\frac{C}{4 \beta} \int_{0}^{L_{0}} \rho_{0}^{2} H^{2} d \xi
$$

Consequently,

$$
\begin{gathered}
\int_{0}^{L_{0}}\left(\rho^{\star}\right)^{2}\left|z_{t}\right|^{2} d \xi+\frac{d}{d t} \int_{0}^{L_{0}} b^{\ell}\left(\rho^{\star}\right)^{2}\left|z_{\xi}\right|^{2} d \xi \leq C\left(\int_{0}^{L_{0}}\left(v^{2} \mathbb{1}_{\mathcal{O}}+h^{2}\right)\left(\rho^{\star}\right)^{2} d \xi\right. \\
\left.+\int_{0}^{L_{0}} \hat{\rho}^{2}\left|z_{\xi}\right|^{2} d \xi+\int_{0}^{L_{0}}\left(\rho^{\star}\right)^{2}\left|z_{\xi}\right|^{2} d \xi\right) .
\end{gathered}
$$

Integrating in time and using (3.19), (3.15) and Poincaré's inequality, we find that

$$
\begin{aligned}
\int_{0}^{t} \int_{0}^{L_{0}} & \left(\rho^{\star}\right)^{2}\left|z_{t}\right|^{2} d \xi d s+\int_{0}^{L_{0}}\left(\rho^{\star}\right)^{2}\left|z_{\xi}\right|^{2} d \xi \\
& \leq C\left(\left\|u_{0}\right\|_{H_{0}^{1}\left(0, L_{0}\right)}^{2}+\|z\|_{L^{2}\left(\rho^{2} ; Q_{L_{0}}\right)}^{2}+\|v\|_{L^{2}\left(\rho_{0}^{2} ; \mathcal{O}\right)}^{2}\right. \\
& \left.+\|h\|_{L^{2}\left(\rho_{0}^{2} ; Q_{L_{0}}\right)}^{2}+\int_{0}^{t} \int_{0}^{L_{0}}\left(\rho^{\star}\right)^{2}\left|z_{\xi}\right|^{2} d \xi d s\right) .
\end{aligned}
$$

Finally, from Gronwall's inequality, taking limits as $t$ goes to $T$, we deduce (3.20).

- Proof of (3.21): Now, let us multiply the first line of (3.1) by $-\left(\rho^{\star}\right)^{2} z_{\xi \xi}$ and let us integrate in space. We see that

$$
\begin{aligned}
\frac{1}{2} \frac{d}{d t} \int_{0}^{L_{0}}\left(\rho^{\star}\right)^{2}\left|z_{\xi}\right|^{2} d \xi & +\int_{0}^{L_{0}} b^{\ell}\left(\rho^{\star}\right)^{2}\left|z_{\xi \xi}\right|^{2} d \xi \\
& =-\int_{0}^{L_{0}}\left(v \mathbb{1}_{\mathcal{O}}+h\right)\left(\rho^{\star}\right)^{2} z_{\xi \xi} d \xi-2 \int_{0}^{L_{0}} \rho^{\star} \rho_{\xi}^{\star} z_{\xi} z_{t} d \xi \\
& +\int_{0}^{L_{0}} \rho^{\star} \rho_{t}^{\star}\left|z_{\xi}\right|^{2} d \xi+\int_{0}^{L_{0}} a^{\ell}\left(\rho^{\star}\right)^{2} z_{\xi} z_{\xi \xi} d \xi .
\end{aligned}
$$

Using (3.19) and (3.20) and arguing in a very smilar way, we get:

$$
\begin{aligned}
\int_{0}^{L_{0}}\left(\rho^{\star}\right)^{2}\left|z_{\xi}\right|^{2} d \xi & +\int_{0}^{t} \int_{0}^{L_{0}}\left(\rho^{\star}\right)^{2}\left|z_{\xi \xi}\right|^{2} d \xi d s \\
& \leq C\left(\left\|u_{0}\right\|_{H_{0}^{1}\left(0, L_{0}\right)}^{2}+\|h\|_{L^{2}\left(\rho_{0}^{2} ; Q_{L_{0}}\right)}^{2}+\int_{0}^{t} \int_{0}^{L_{0}} \hat{\rho}^{2}\left|z_{\xi}\right|^{2} d \xi d s\right) .
\end{aligned}
$$

Again, from Grönwall's inequality, (3.21) is easily found.

We end this section by pointing out that the ideas used throughout it can be adapted to solve control problems under more general constraints (for instance, a finite number of onedimensional restrictions as (3.2)). In fact, the key point here is that $\widehat{P}$, the projection induced by the constraints, must to be compact. 


\section{Proof of the Main Result}

Let us begin this section by mentioning that in order to prove our main result, equation (1.1) can be allowed to evolve, during a short time interval, without the influence of the control (i.e $v \equiv 0)$. Then, from regularity of the Stefan problem, in spite of Theorem 1.1 been stated with initial data in $H_{0}^{1}\left(0, L_{0}\right)$, to prove this result we can assume, without loss of generality, much more regularity for the initial data.

Let $\mathcal{X}$ be the space of triplets $(z, v, \ell)$ satisfying:

(i) $z \in L^{2}\left(\rho^{2} ; Q_{L_{0}}\right)$ and

a) $z(\cdot, 0) \in C_{0}^{2+\alpha}\left(\left[0, L_{0}\right]\right), z \in \mathcal{C}^{2+\alpha, 1+\alpha / 2}\left(\left[L_{*}, L_{0}\right] \times[0, T]\right) .{ }^{3}$

b) $z(0, t)=z\left(L_{0}, t\right)=0$ for $t \in(0, T)$.

c) $M^{L_{0}} z \in L^{2}\left(\rho_{0}^{2} ; Q_{L_{0}}\right)$.

(ii) $v \in L^{2}\left(\rho_{0}^{2} ; \mathcal{O}\right)$.

(iii) $\ell \in C^{1+\alpha / 2}([0, T])$ and
a) $\ell(t)=-\int_{0}^{t} z_{\xi}\left(L_{0}, s\right) d s$.
b) $\ell(T)=0$.

On the other hand, let us set $\mathcal{Y}:=F \times C_{0}^{2+\alpha}\left(\left[0, L_{0}\right]\right)$, where

$$
F:=\left\{h \in L^{2}\left(\rho_{0}^{2} ; Q_{L_{0}}\right): h \in \mathcal{C}^{\alpha, \alpha / 2}\left(\left[L_{*}, L_{0}\right] \times[0, T]\right)\right\} .
$$

It is clear that $\mathcal{X}$ and $\mathcal{Y}$ are Banach spaces for the norms

$$
\begin{gathered}
\|(z, v, \ell)\|_{\mathcal{X}}^{2}:=\|z\|_{L^{2}\left(\rho^{2} ; Q_{L_{0}}\right)}^{2}+\left\|M^{L_{0}} z\right\|_{L^{2}\left(\rho_{0}^{2} ; Q_{L_{0}}\right)}^{2}+\|z(\cdot, 0)\|_{C_{0}^{2+\alpha}\left(\left[0, L_{0}\right]\right)}^{2} \\
+\|z\|_{\mathcal{C}^{2+\alpha, 1+\alpha / 2}\left(\left[L_{*}, L_{0}\right] \times[0, T]\right)}^{2}+\|v\|_{L^{2}\left(\rho_{0}^{2} ; \mathcal{O}\right)}^{2}+\|\ell\|_{C^{1+\alpha / 2}([0, T])}^{2}, \\
\left\|\left(h, u_{0}\right)\right\|_{\mathcal{Y}}^{2}:=\|h\|_{L^{2}\left(\rho_{0}^{2} ; Q_{L_{0}}\right)}^{2}+\|h\|_{\mathcal{C}^{\alpha, \alpha / 2}\left(\left[L_{*}, L_{0}\right] \times[0, T]\right)}^{2}+\left\|u_{0}\right\|_{C_{0}^{2+\alpha}([0, T])}^{2} .
\end{gathered}
$$

Lemma 4.1. For each $n \geq 0$, there exists $d_{n}>0$ such that, for any $(z, v, \ell) \in \mathcal{X}$, one has:

$$
\left\|\ell m^{-(n+1 / 2)}\right\|_{\infty} \leq d_{n}\|(z, v, \ell)\|_{\mathcal{X}}
$$

(recall the definition of $m$ in (3.3)).

Proof. In view of the embedding of $H^{1}(a, b)$ in $C^{0}[a, b]$, we have

$$
\left|\ell^{\prime}(t)\right|^{2} \leq\left|z_{\xi}\left(L_{0}, t\right)\right|^{2} \leq C \int_{L_{*}}^{L_{0}}\left(\left|z_{\xi}(\xi, t)\right|^{2}+\left|z_{\xi \xi}(\xi, t)\right|^{2}\right) d \xi .
$$

Let us introduce $\bar{\rho}(t):=\exp \left\{\frac{\kappa}{T-t}\right\}$, where $\kappa$ is chosen such that $0<2 \kappa<\min _{x \in\left[0, L_{0}\right]}\{\tilde{\alpha}(x)\}$ and $\bar{\rho} \leq \rho^{1 / 2}$. Then

$$
\begin{aligned}
\int_{0}^{T} \bar{\rho}^{2}\left|\ell^{\prime}(s)\right|^{2} d s & \leq C \iint_{Q_{L_{0}}} \rho\left(\left|z_{\xi}(\xi, t)\right|^{2}+\left|z_{\xi \xi}(\xi, t)\right|^{2}\right) d \xi d t \\
& \leq C\left(\int_{Q_{L_{0}}} \rho^{2} m^{2}\left|z_{\xi}(\xi, t)\right|^{2} d \xi d t+\int_{Q_{L_{0}}} \rho^{2} m^{4}\left|z_{\xi \xi}(\xi, t)\right|^{2} d \xi d t\right) \\
& \leq C\|(z, v, \ell)\|_{\mathcal{X}}^{2}
\end{aligned}
$$

(the last inequality comes from the estimates (3.19) and (3.21)).

Since by hypothesis $\ell(T)=0$, we can write that $\ell(t)=-\int_{t}^{T} \ell^{\prime}(s) d s$. For each $n$, there exists $c_{n}>0$ such that $(T-t)^{-n} \leq c_{n} \bar{\rho}(t)$ for all $t \in[0, T)$. Therefore, using Schwarz's inequality, we see that

$$
\begin{aligned}
|\ell(t)| & \leq\left(\int_{t}^{T}(T-t)^{-2 n}\left|\ell^{\prime}(t)\right|^{2} d s\right)^{1 / 2}\left(\int_{t}^{T}(T-t)^{2 n} d s\right)^{1 / 2} \\
& \leq \sqrt{\frac{C c_{2 n}}{2 n+1}}(T-t)^{n+1 / 2}\|(z, v, \ell)\|_{\mathcal{X}} .
\end{aligned}
$$

From the definition of $m$, we also have that

$$
|\ell(t)| \leq \sqrt{\frac{C c_{2 n}}{2 n+1}}(2 / T)^{n+1 / 2} m(t)^{n+1 / 2}\|(z, v, \ell)\|_{\mathcal{X}},
$$

for all $t \in[T / 2, T]$. Finally, using again Schwarz's inequality and the fact that $m$ is a constant times $T^{2} / 4$ in $[0, T / 2]$, we conclude that

$$
|\ell(t)| \leq 2^{2 n+1} T^{-2 n} m(t)^{n+1 / 2}\left\|\ell^{\prime}\right\|_{\infty}
$$

\footnotetext{
${ }^{3}$ The Hölder spaces $\mathcal{C}^{2+\alpha, 1+\alpha / 2}$ are defined in the Appendix (see Section 6).
} 
for all $t \in[0, T / 2]$.

Let us consider the open set

$$
D:=\left\{(z, v, \ell) \in \mathcal{X}: \ell(t)>L_{\star}-L_{0} \text { for all } t \in[0, T]\right\}
$$

and define $\mathcal{F}: D \subset \mathcal{X} \rightarrow \mathcal{Y}$ by

$$
\mathcal{F}(z, v, \ell)=\left(M^{\ell+L_{0}} z-v \mathbb{1}_{\mathcal{O}}, z(0)\right) .
$$

Remark 4.2. According to item iii) in the definition of $\mathcal{X}, \mathcal{F}$ is a function of $z$ and $v$. Nevertheless, for simplicity of notation, we will keep the three variables $z, v$ and $\ell$ as arguments of $\mathcal{F}$.

Lemma 4.3. The mapping $\mathcal{F}: D \subset \mathcal{X} \mapsto \mathcal{Y}$ is well defined.

Proof. Suppose that $\left(h, z_{0}\right)=F(z, v, \ell)$ for some $(z, v, \ell) \in \mathcal{X}$. It is clear, from the definition of $\mathcal{X}$, that $h \in \mathcal{C}^{\alpha, \alpha / 2}\left(\left[L_{*}, L_{0}\right] \times[0, T]\right)$ and $z_{0} \in C_{0}^{2+\alpha}\left(\left[0, L_{0}\right]\right)$. Therefore, it only remains to show that $M^{\ell+L_{0}} z \in L^{2}\left(\rho_{0}^{2} ; Q_{L_{0}}\right)$, for which it is enough to verify

$$
\begin{aligned}
& \left(a^{\ell+L_{0}}-a^{L_{0}}\right) z_{\xi} \in L^{2}\left(\rho_{0}^{2} ; Q_{L_{0}}\right) . \\
& \left(b^{\ell+L_{0}}-b^{L_{0}}\right) z_{\xi \xi} \in L^{2}\left(\rho_{0}^{2} ; Q_{L_{0}}\right) .
\end{aligned}
$$

In view of the a priori estimates (3.19), (3.21) and the fact that $\left|a^{\ell+L_{0}}-a^{L_{0}}\right| \leq c_{0}|\ell|+c_{1}\left|\ell^{\prime}\right|$ and $\left|b^{\ell+L_{0}}-b^{L_{0}}\right| \leq c_{2}|\ell|$, it wil be enough to check that $m(t)\left(|\ell(t)|+\left|\ell^{\prime}(t)\right|\right)$ and $m^{-1}(t)(|\ell(t)|)$ are uniformly bounded on $[0, T]$.

The first assertion is immediately verified and the second one is a consequence of Lemma 4.1.

It is exactly at this point that we can see that the constraint (3.2) is needed. In fact, since $m(T)=0$, we must to impose $\ell(T)=0$ if we want that $m^{-1}(t)(|\ell(t)|)$ be bounded in $[0, T]$. Although, in principle, this can be viewed as a technical restriction, it makes physical sense if we interpret (1.1) as a model for a semi-infinite one-dimensional block of ice, initially at melting temperature $u=0$ for $x \in\left[L_{0}, \infty\right)$ and $u=u_{0}$ for $x \in\left[0, L_{0}\right]$, that we want to drive to temperature $u=0$ for all $x \in[0, \infty)$ at time $T$.

Lemma 4.4. The mapping $\mathcal{F}: D \subset \mathcal{X} \rightarrow \mathcal{Y}$ is continuously differentiable.

Proof. In view of the results in the previous sections, we see that the derivative of $\mathcal{F}$ at any point $(z, v, \ell) \in D$ is given by

$$
\mathcal{F}^{\prime}(z, v, \ell)(\tilde{z}, \tilde{v}, \tilde{\ell})=\left(d a(\ell, \tilde{\ell}) z_{\xi}-d b(\ell, \tilde{\ell}) z_{\xi \xi}+M^{L_{0}+\ell} \tilde{z}-\tilde{v} \mathbb{1}_{\mathcal{O}}, \tilde{z}(0)\right),
$$

for any $(\tilde{z}, \tilde{v}, \tilde{\ell})$ in $\mathcal{X}$. The expressions $d a(\ell, \tilde{\ell})$ and $d b(\ell, \tilde{\ell})$ appearing above are respectively defined in (6.1) and (6.2) and can be written in the form

$$
d a(\ell, \tilde{\ell})(\xi, t)=\hat{a}\left(\ell(t), \ell^{\prime}(t), \xi\right) \cdot\left(\tilde{\ell}(t), \tilde{\ell}^{\prime}(t)\right) \quad \text { and } \quad d b(\ell, \tilde{\ell})(\xi, t)=\hat{b}(\ell(t), \xi) \tilde{\ell}(t),
$$

where $\hat{a}$ and $\hat{b}$ are smooth functions.

Now, we proceed to verify that $\mathcal{F}^{\prime}: D \subset \mathcal{X} \rightarrow \mathcal{L}(\mathcal{X}, \mathcal{Y})$ is a continuous function. To this end, it is enough to prove that for any $(z, v, \ell) \in D$, if $\left(z_{n}, v_{n}, \ell_{n}\right) \rightarrow(z, v, \ell)$ in $\mathcal{X}$, there exists a sequence $\delta_{n} \rightarrow 0$ such that

$$
\left\|\left(\mathcal{F}^{\prime}\left(z_{n}, v_{n}, \ell_{n}\right)-\mathcal{F}^{\prime}(z, v, \ell)\right)(\tilde{z}, \tilde{v}, \tilde{\ell})\right\|_{\mathcal{Y}}^{2} \leq \delta_{n}\|(\tilde{z}, \tilde{v}, \tilde{\ell})\|_{\mathcal{X}}^{2} .
$$

Note that the left hand side of this last inequality corresponds to

$$
\left\|\left(h_{n}-h\right)\right\|_{L^{2}\left(\rho_{0}^{2} ; Q_{L_{0}}\right)}^{2}+\left\|\left(h_{n}-h\right)\right\|_{\mathcal{C}^{\alpha, \alpha / 2}\left(\left[L_{*}, L_{0}\right] \times[0, T]\right)}^{2},
$$

where $h_{n}=d a\left(\ell_{n}, \tilde{\ell}\right) z_{\xi}-d b\left(\ell_{n}, \tilde{\ell}\right) z_{\xi \xi}+M^{L_{0}+\ell_{n}} \tilde{z}$ and $h=d a(\ell, \tilde{\ell}) z_{\xi}-d b(\ell, \tilde{\ell}) z_{\xi \xi}+M^{L_{0}+\ell} \tilde{z}$. Therefore, we have to prove the existence of a sequence $\delta_{n} \rightarrow 0$ such that

$$
\left\|\left(h_{n}-h\right)\right\|_{L^{2}\left(\rho_{0}^{2} ; Q_{L_{0}}\right)}^{2} \leq \delta_{n}\|(\tilde{z}, \tilde{v}, \tilde{\ell})\|_{\mathcal{X}}^{2} \text { and }\left\|\left(h_{n}-h\right)\right\|_{\mathcal{C}^{\alpha, \alpha / 2}\left(\left[L_{*}, L_{0}\right] \times[0, T]\right)}^{2} \leq \delta_{n}\|(\tilde{z}, \tilde{v}, \tilde{\ell})\|_{\mathcal{X}}^{2}
$$


The first inequality follows from the regularity of $\hat{a}$ and $\hat{b}$ in (4.5), the a priori estimates (3.19) and (3.21) and Lemma 4.1, by noting that $\left\|\left(h_{n}-h\right)\right\|_{L^{2}\left(\rho_{0}^{2} ; Q_{L_{0}}\right)}^{2}$ can be bounded from above by 6 times

$$
\begin{aligned}
\delta_{1 n} C_{1}\|\tilde{\ell}\|_{C^{1}[0, T]}^{2}+\delta_{2 n} C_{2}\|\tilde{\ell}\|_{C^{1}[0, T]}^{2} & +\left(\delta_{3 n} C_{3}+\delta_{4 n} C_{4}\right)\|\tilde{\ell}(t) / \sqrt{m(t)}\|_{\infty}^{2} \\
& \left.+\frac{T^{2}}{4} \delta_{5 n}\left\|\tilde{z}_{\xi}\right\|_{L^{2}\left(\hat{\rho}^{2} ; Q_{L_{0}}\right)}^{2}+\delta_{6 n}\left\|\hat{b}\left(L_{0}+\ell\right)\right\|_{\infty}^{2}\left\|\tilde{z}_{\xi \xi}\right\|_{L^{2}\left(\left(\rho^{\star}\right)^{2} ; Q_{L_{0}}\right.}^{2}\right)
\end{aligned}
$$

where,

$$
\begin{array}{rlrl}
\delta_{1 n} & =\sup _{(\xi, t) \in \overline{Q_{L_{0}}}}\left|\hat{a}\left(\ell_{n}(t), \ell_{n}^{\prime}(t), \xi\right)-\hat{a}\left(\ell(t), \ell^{\prime}(t), \xi\right)\right|^{2} & C_{1}=\sup _{n \in \mathbb{N}}\left\|\left(z_{n}\right)_{\xi}\right\|_{L^{2}\left(\rho_{0}^{2} ; Q_{L_{0}}\right)}^{2} \\
\delta_{2 n}=\left\|\left(z_{n}-z\right)_{\xi}\right\|_{L^{2}\left(\rho_{0}^{2} ; Q_{L_{0}}\right)} & C_{2}=\sup _{(\xi, t) \in \overline{Q_{L_{0}}}}\left|\hat{a}\left(\ell(t), \ell^{\prime}(t), \xi\right)\right|^{2} \\
\delta_{3 n}=\sup _{(\xi, t) \in \overline{Q_{L_{0}}}}\left|\hat{b}\left(\ell_{n}(t), \xi\right)-\hat{b}(\ell(t), \xi)\right|^{2} & C_{3}=\sup _{n \in \mathbb{N}}\left\|\left(z_{n}\right)_{\xi \xi}\right\|_{L^{2}\left(\left(\rho^{\star}\right)^{2} ; Q_{L_{0}}\right)}^{2} \\
\delta_{4 n}=\left\|\left(z_{n}-z\right)_{\xi \xi}\right\|_{L^{2}\left(\left(\rho^{\star}\right)^{2} ; Q_{L_{0}}\right)}^{2} & C_{4}=\sup _{(\xi, t) \in \overline{Q_{L_{0}}}}|\hat{b}(\ell(t), \xi)|^{2} \\
\delta_{5 n}=\sup _{(\xi, t) \in \overline{Q_{L_{0}}}}\left|a^{L_{0}+\ell_{n}}(\xi, t)-a^{L_{0}+\ell}(\xi, t)\right| & C_{6}=\sup _{0 \leq \theta \leq 1}\left\|\left(L_{0}+\ell\right)+\theta\left(\ell-\ell_{n}\right)\right\|_{\infty}^{2} \\
\delta_{6 n}=\left\|\left(\ell-\ell_{n}\right)(t) / \sqrt{m(t)}\right\|_{\infty}^{2} . &
\end{array}
$$

The second inequality can be obtained in a similar way.

Lemma 4.5. $\mathcal{F}^{\prime}(0,0,0): \mathcal{X} \rightarrow \mathcal{Y}$ is surjective.

Proof. We have to show that, for every $\left(h, u_{0}\right) \in \mathcal{Y}$, there exists $(z, v, \ell) \in \mathcal{X}$ such that:

(1) $M^{L_{0}} z-v \mathbb{1}_{\mathcal{O}}=h$.

(2) $z(0)=u_{0}$.

Since $h \in L^{2}\left(\rho_{0}^{2} ; Q_{L_{0}}\right)$ and $u_{0} \in C_{0}^{2+\alpha}\left(\left[0, L_{0}\right]\right)$ (in particular to $\left.H_{0}^{1}\left(\left(0, L_{0}\right)\right)\right)$, and in view of Theorem 3.3, there exists a pair of functions $(z, v)$ satisfying (a) and (b) above, together with conditions (i) b), (i) c) and (ii) in the definition of $\mathcal{X}$. Moreover, defining

$$
\ell(t)=-\int_{0}^{t} \tilde{z}_{\xi}\left(L_{0}, s\right) d s
$$

it is also guaranteed by Theorem 3.3 that $\ell(T)=0$.

The proof of the Hölder regularity of $z$ and $\ell$ needed to fulfill the definition of $\mathcal{X}$ will be divided in two steps. First, since $u_{0} \in C_{0}^{2+\alpha}\left(\left[0, L_{0}\right]\right)$ and $h \in \mathcal{C}^{\alpha, \alpha / 2}\left(\left[L_{*}, L_{0}\right] \times[0, T]\right)$, Theorem 6.1 implies that $z \in \mathcal{C}^{2+\alpha, 1+\alpha / 2}\left(\left[L_{*}, L_{0}\right] \times[0, T]\right)$. Secondly, applying Theorem 6.2 to the problem

$$
\begin{cases}M^{L_{0}} w(\xi, t)=h(\xi, t) & \text { in } \quad\left(L_{*}, L_{0}\right) \times[0, T], \\ w(0, t)=z\left(L_{*}, t\right) w\left(L_{0}, t\right)=0 & \text { for } t \in(0, T), \\ w(\xi, 0)=u_{0}(\xi) & \text { for } \xi \in\left(L_{*}, L_{0}\right),\end{cases}
$$

we can conclude that $z$ also satisfies condition (i) a) in the definition of $\mathcal{X}$. Finally, the desired regularity for $\ell$ defined above follows from (i) a).

Remark 4.6. Taking into account the formulation (1.5) of the null controllability problem and the fact that $\mathcal{F}^{\prime}(0,0,0)$ is surjective, we can introduce, as in [4], the following quasi-Newton iterates

$$
\left(z^{n+1}, v^{n+1}, \ell^{n+1}\right)=\left(z^{n}, v^{n}, \ell^{n}\right)-\mathcal{G}\left(\mathcal{F}\left(z^{n}, v^{n}, \ell^{n}\right)-\left(h, z_{0}\right)\right), \quad n \geq 0 .
$$

Here, $\mathcal{G}$ is an inverse to $\mathcal{F}^{\prime}(0,0,0)$. It can be proved that, if the starting triplet $\left(z^{0}, v^{0}, \ell^{0}\right)$ is close enough to $(0,0,0)$, the sequence $\left\{\left(z^{n}, v^{n}, \ell^{n}\right)\right\}$ converges as $n \rightarrow+\infty$ to a solution to (1.5). 


\section{Some AdDitional COMments}

Note that, instead of (1.1)-(1.2), we can also consider the following free-boundary system, where the PDE is semilinear:

$$
\begin{cases}u_{t}-u_{x x}+f(u)=v \mathbb{1}_{\mathcal{O}} & \text { in } \quad Q_{\ell}, \\ u(0, t)=u(\ell(t), t)=0 & \text { for } t \in(0, T), \\ u(x, 0)=u_{0}(x) & \text { for } \quad x \in\left(0, L_{0}\right), \\ \ell^{\prime}(t)=-\frac{1}{k} u_{x}(\ell(t), t) & \text { for } t \in(0, T), \\ \ell(0)=L_{0}, & \\ u(x, T)=0, & x \in(0, \ell(T)) .\end{cases}
$$

Thus, if we assume (for instance) that $f: \mathbb{R} \mapsto \mathbb{R}$ is globally Lipschitz-continuous and $f(0)=0$, the arguments in Sections 2-4 can be adapted to prove a result similar to Theorem 1.1.

On the other hand, it is completely meaningful to consider a problem similar to (1.1)-(1.2) in higher dimensions. Thus, $\Omega_{0} \subset \mathbb{R}^{N}$ be a bounded connected open set, let $G \Subset \Omega_{0}$ be a non-empty open subset, let us assume that $y_{0}: \Omega_{0} \mapsto \mathbb{R}$ and $T>0$ are given and let us consider the system

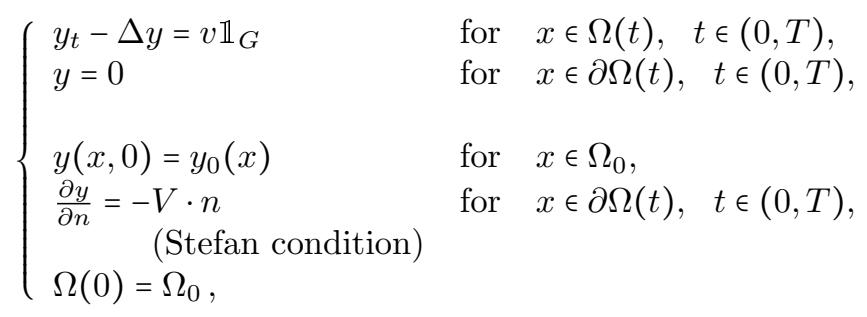

where $n=n(x, t)$ denotes the outward unit normal and $V=V(x, t)$ is the speed at which $\Omega(t)$ expands at the points $x \in \partial \Omega(t)$. Now, the null controllability problem is to find $y, v$ and $\{\Omega(t)\}_{t \in[0, T]}$ such that one has (5.3) and, moreover,

$$
y(x, T)=0, \quad x \in \Omega(T) .
$$

In a forthcoming paper, we will use techniques similar to those in the previous sections to prove a local controllability result for (5.3)-(5.4).

\section{Appendix: Some technical Results}

6.1. Differentiability of $M^{\ell}$. Now we proceed to study the applications which make correspond to any $\ell \in C^{1}([0, T])$ the coefficients of the differential operator $M^{\ell}$, namely, $\ell \mapsto a^{\ell}$ and $\ell \mapsto b^{\ell}$. From now on, we will use the notations $a^{\ell}$ and $a(\ell)$ indistinctly.

Let us define $d a(\ell, \tilde{\ell})(\xi, t):=\left.\frac{d}{d \epsilon} a^{\ell+\epsilon \tilde{\ell}}(\xi, t)\right|_{\epsilon=0}$. Using the chain rule we can see that $d a(\ell, \tilde{\ell})(\xi, t)$ is equal to

$$
\begin{aligned}
H_{y}(x(\xi, t), \ell(t)) \tilde{\ell}^{\prime}(t)+\nabla H_{y}( & x(\xi, t), \ell(t)) \cdot\left(H_{y}(x(\xi, t), \ell(t)), 1\right) \ell^{\prime}(t) \tilde{\ell}(t) \\
& +\nabla H_{x x}(x(\xi, t), \ell(t)) \cdot\left(H_{y}(x(\xi, t), \ell(t)), 1\right) \tilde{\ell}(t),
\end{aligned}
$$

where $x(\xi, t)$ denotes $H^{-1}(\xi, \ell(t))$. Analogously, we obtain

$$
d b(\ell, \tilde{\ell})(\xi, t)=2 H_{x}(x(\xi, t), \ell(t)) \nabla H_{x}(x(\xi, t), \ell(t)) \cdot\left(H_{y}(x(\xi, t), \ell(t)), 1\right) \tilde{\ell}(t) .
$$

Now we proceed to verify that $d a(\ell, \tilde{\ell})$ (resp. $d a_{\xi}(\ell, \tilde{\ell})$ and $\left.d b(\ell, \tilde{\ell})\right)$ is in fact the Gâteauxderivative of $a$ (resp. $b$ ) at $\ell$ along the direction $\tilde{\ell}$. Here, we deal with $b^{\ell}=H_{x}^{2}\left(H^{-1}(\xi, \ell(t)), \ell(t)\right)$. The other cases can be handled in a similar way.

Let us fix $x=H^{-1}(\xi, \ell(t)), y=\ell(t), h_{1}=H^{-1}(\xi, \ell(t)+\epsilon \tilde{\ell}(t))-H^{-1}(\xi, \ell(t))$ and $h_{2}=\epsilon \tilde{\ell}(t)$. Using the Taylor's first order expansion of $H_{x}^{2}$, we obtain:

$$
\left|b^{\ell+\epsilon \tilde{\ell}}(\xi, t)-b^{\ell}(\xi, t)-\nabla H_{x}^{2}(x, y) \cdot\left(h_{1}, h_{2}\right)\right| \leq C_{1}\left\|\left(h_{1}, h_{2}\right)\right\|^{2},
$$

where $C_{1}$ can be taken as the supremum of $\left\|\operatorname{Hess}\left(H_{x}^{2}\right)\right\|$ on a fixed domain, says $\overline{Q_{\ell+\mid \tilde{\ell}}}$. On the other hand

$$
\left|H^{-1}(\xi, \ell(t)+\epsilon \tilde{\ell}(t))-H^{-1}(\xi, \ell(t))-\nabla H^{-1}(\xi, \ell(t)) \cdot(0, \epsilon \tilde{\ell}(t))\right| \leq C_{2}|\epsilon \tilde{\ell}(t)|^{2}
$$


where $C_{2}:=\sup _{(z, w) \in H^{-1}\left(\overline{Q_{\ell+|\tilde{\mid}|}}\right)}\left\|\operatorname{Hess}\left(H^{-1}(z, w)\right)\right\|$. Therefore,

$$
\begin{aligned}
\left|b^{\ell+\epsilon \tilde{\ell}}(\xi, t)-b^{\ell}(\xi, t)-\epsilon d b(\ell, \tilde{\ell})\right| & \leq\left|b^{\ell+\epsilon \tilde{\ell}}(\xi, t)-b^{\ell}(\xi, t)-\nabla H_{x}^{2}(x, y) \cdot\left(h_{1}, h_{2}\right)\right| \\
+ & \left|\nabla H_{x}^{2}(x, y) \cdot\left(h_{1}-\epsilon H_{y}(x, y) \tilde{\ell}(t), 0\right)\right|
\end{aligned}
$$

and thus

$$
\left|\frac{b^{\ell+\epsilon \tilde{\ell}}(\xi, t)-b^{\ell}(\xi, t)}{\epsilon}-d b(\ell, \tilde{\ell})\right| \leq 3 C \epsilon|\tilde{\ell}(t)|^{2}
$$

where we can take

$$
C=C_{1} C_{2} \sup _{(x, t) \in \overline{Q_{\ell+|\tilde{\ell}|}}}\left\|\nabla H_{x}^{2}(x, t)\right\| .
$$

6.2. Hölder regularity of parabolic equations. Given a connected open set $\Omega \subset \mathbb{R}^{n}$, consider the cylinder $Q_{T}=\Omega \times(0, T)$ and denote by $S_{T}=\{(x, t): x \in \partial \Omega, t \in[0, T]\}$ its lateral surface. Let $\mathcal{C}^{\alpha, \alpha / 2}\left(\overline{Q_{T}}\right)$ be, for any $\alpha \in(0,1]$, the space of functions $u: \overline{Q_{T}} \mapsto \mathbb{R}$ such that $D_{t}^{r} D_{x}^{s} u$ is:

i) Continuous on $\bar{\Omega}$ for $0 \leq 2 r+s \leq\lfloor\alpha\rfloor$.

ii) $\gamma$-Hölder continuous in space of index $\gamma=\alpha-\lfloor\alpha\rfloor$ for $2 r+s=\lfloor\alpha\rfloor$.

iii) $\gamma$-Hölder continuous in time of index $\gamma=(\alpha-2 r-s) / 2$ for $0<\alpha-2 r-s<2$.

Obviously, $\mathcal{C}^{\alpha, \alpha / 2}\left(\overline{Q_{T}}\right)$ is a Banach space for the norm

$$
\begin{gathered}
\|\cdot\|_{C^{\alpha, \alpha / 2}\left(\overline{Q_{T}}\right)=} \sum_{0 \leq 2 r+s \leq\lfloor\alpha\rfloor}\left\|D_{t}^{r} D_{x}^{s} u\right\|_{\infty}+\sum_{2 r+s=\lfloor\alpha\rfloor}\left\|D_{t}^{r} D_{x}^{s} u\right\|_{C_{x}^{\alpha-\lfloor\alpha\rfloor}\left(\overline{Q_{T}}\right)} \\
+\sum_{0<\alpha-2 r-s<2}\left\|D_{t}^{r} D_{x}^{s} u\right\|_{C_{x}^{(\alpha-2 r-s) / 2}\left(\overline{Q_{T}}\right)},
\end{gathered}
$$

where $\|\cdot\|_{\infty}$ denotes the norm of the uniform convergence and

$$
\|u\|_{C_{x}^{\gamma}\left(\overline{Q_{T}}\right)}=\sup _{(x, t),(y, t) \in \overline{Q_{T}}} \frac{|u(x, t)-u(y, t)|}{|x-y|^{\gamma}}, \quad\|u\|_{C_{t}^{\gamma}\left(\overline{Q_{T}}\right)}=\sup _{(x, s),(x, t) \in \overline{Q_{T}}} \frac{|u(x, s)-u(x, t)|}{|s-t|^{\gamma}} .
$$

Consider the differential operator

$$
\mathcal{L} u=u_{t}-\sum_{i, j=1}^{n} a_{i, j}(x, t) u_{x_{i}, x_{j}}+\sum_{i=1}^{n} a_{i}(x, t) u_{x_{i}}+a(x, t) u .
$$

We will say that the problem

$$
\left\{\begin{array}{l}
\mathcal{L} u=f(x, t) \\
\left.u\right|_{t=0}=\phi(x) \\
\left.u\right|_{S_{T}}=\Phi(x, t)
\end{array}\right.
$$

satisfies the compatibility conditions of order $m \geq 0$ if

$$
\left.u^{(k)}(x)\right|_{x \in S}=\left.\frac{\partial^{k} u}{\partial t^{k}}\right|_{t=0}=\left.\frac{\partial^{k} \Phi}{\partial t^{k}}\right|_{t=0}=\Phi^{(k)}(x) \text { for } k=0, \cdots, m .
$$

The next two results are classical and well known (see Theorems III.12.2 and IV.5.2 of [22]):

Theorem 6.1. Suppose $u \in W_{q}^{2,1}\left(Q_{T}\right)$ is a generalized solution of $\mathcal{L} u=f(x, t)$. If $f$ and the coefficients of the operator $\mathcal{L}$ belong to $\mathcal{C}^{\alpha, \alpha / 2}\left(Q_{T}\right)$, then u belongs to $\mathcal{C}^{2+\alpha, 1+\alpha / 2}\left(Q_{T}\right)$.

See also the comments in [22], p. 223, below the statement of Theorem III.12.2.

Theorem 6.2. Suppose that $\alpha>0$, the coefficients of the operator $\mathcal{L}$ belong to $\mathcal{C}^{\alpha, \alpha / 2}\left(\overline{Q_{T}}\right)$ and the boundary $S$ is sufficiently regular (more precisely, of class $\mathcal{C}^{\alpha+2}$ ). Then, for any $f \in$ $\mathcal{C}^{\alpha, \alpha / 2}\left(\overline{Q_{T}}\right), \phi \in \mathcal{C}^{\alpha+2}(\bar{\Omega})$ and $\Phi \in \mathcal{C}^{\alpha+2, \alpha / 2+1}\left(\overline{S_{T}}\right)$ satisfying the compatibility conditions of order $\lceil\alpha / 2\rceil,(6.3)$ possesses exactly one solution in $\mathcal{C}^{\alpha+2, \alpha / 2+1}\left(\overline{Q_{T}}\right)$.

\section{ACKLOWLedgements}

E. F-C. was partially supported by MINECO (Spain), Grant MTM2013-41286-P. We would like to express our thanks to the anonymous referee for their helpful comments. 


\section{REFERENCES}

[1] V. M. Alekseev, V. M. Tikhomirov, S. V. Fomin. Optimal Control. Consultants Bureau. New York, 1987.

[2] Aronson, D.G., Some properties of the interface for a gas flow in porous media, in "Free Boundary Problems: Theory and Applications, Volume I", (Fasano A. and Primicerio, M. eds.), Research Notes Math., no. 78, Pitman, London, 1983.

[3] Avalos, G., Lasiecka, I., Boundary controllability of thermoelastic plates via the free boundary conditions, SIAM J. Control Optim. 38 (2000), no. 2, 337-383 (electronic).

[4] Clark, H.R., Fernández-Cara, E., Limaco, J., Medeiros, L.A., Theoretical and numerical local null controllability for a parabolic system with local and nonlocal nonlinearities, Applied Mathematics and Computation, 223, 483-505 (2013).

[5] Doubova, A., Fernández-Cara, E., Some control results for sim-plified one-dimensional models of fluid-solid interaction, Math. Models Methods Appl. Sci. 15 (2005), no. 5, 783-824.

[6] Doubova, A., Fernández-Cara, E., González-Burgos, M., Zuazua, E., On the controllability of parabolic systems with a nonlinear term involving the state and the gradient, SIAM J. Control Optim. 41 (2002), no. 3, 798-819.

[7] Fabre, C., Puel, J. P., Zuazua, E., Approximate controllability of the semilinear heat equation, Proc. Roy. Soc. Edinburgh Sect. A 125 (1) (1995) 31-61.

[8] Fasano, A., Mathematical models of some diffusive processes with free boundaries, MAT, Series A: Mathematical Conferences, Seminars and Papers, 11, Universidad Austral, Rosario, 2005.

[9] Fasano, A., Primicerio, General free-boundary problems for the heat equation, I, J. Math. Anal. Appl. 57 (1977), 694-723.

[10] Fattorini, H.O., Russell, D.L., Exact controllability theorems for linear parabolic equations in one space dimension, Arch. Rational Mech. Anal. 43 (1971), no. 4, 272-292.

[11] Fernández-Cara, E., Guerrero, S., Global Carleman Inequalities for parabolic systems and applications to controllability, SIAM J. Control Optimiz. 45, no. 4 (2006) pp. 1395-1446.

[12] E. Fernández-Cara, J. Límaco, S.B Menezes. On the controllability of a free-boundary problem for the $1 D$ heat equation. Systems \& Control Letters, 87: 29-35, 2016

[13] Fernández-Cara, E., Zuazua, E., The cost of approximate controllability for heat equations: the linear case, Adv. Differential Equations, 5(2000), no. 4-6, 465-514.

[14] Friedman, A., Partial differential equations of parabolic type, Prentice-Hall, Inc., Englewood Cliffs, N.J., 1964.

[15] Friedman, A., Variational principles and free-boundary problems, John Wiley \& Sons, Inc., New York, 1982.

[16] Friedman, A. (Ed.), Tutorials in mathematical biosciences, III. Cell cycle, proliferation, and cancer, Lecture Notes in Mathematics, 1872, Mathematical Biosciences Subseries, Springer-Verlag, Berlin, 2006.

[17] Friedman, A., PDE problems arising in mathematical biology, Netw. Heterog. Media 7 (2012), no. 4, 691703.

[18] Fursikov, A. V., Imanuvilov, O.Yu., Controllability of evolution equations, Lectures Notes Series, Vol. 34, National University, RIM, Seoul, South Korea, 1996.

[19] Gupta, S.C., The classical Stefan problem. Basic concepts, modelling and analysis, Elsevier Science B.V., Amsterdam, 2003.

[20] Hansen, S.W., Imanuvilov, O.Yu, Exact controllability of a multilayer Rao-Nakra plate with free boundary conditions, Math. Control Related Fields 1 (2011), no. 2, 189-230.

[21] Hermans, A. J., Water waves and ship hydrodynamics. An introduction, 2nd edition, Springer, Dordrecht, 2011.

[22] O.A. Ladyzenskaja, V.A. Solonnikov, N.N. Ural'ceva. Linear and quasilinear equations of parabolic type. Translations of Mathematical Monographs, American Mathematical Society, Providence, R.I. 1968.

[23] Lebeau, G., Robbiano, L., Contrôle exact de l'équation de la chaleur, Comm. Partial Differential Equations 20 (1995), no. 1-2, 335-356.

[24] Liu, Y., Takahashi, T., Tucsnak, M., Single input control-llability of a simplified fluid-structure interaction model, ESAIM Control Optim. Calc. Var. 19 (2013), no. 1, 20-42.

[25] Mercan, M., Nakoulima, O., Control of Stackelberg for a two-stroke problem, Dyn. Contin. Discrete Impuls. Syst. Ser. B Appl. Algorithms 22 (2015), no. 6, 441-463.

[26] Nakoulima, O., Contrôlabilité à zéro avec contraintes sur le contrôle, C. R. Math. Acad. Sci. Paris 339 (2004), no. 6, 405-410

[27] Puel, J.-P., Yamamoto, M., On a global estimate in a linear inverse hyperbolic problem,Inverse Problems 12 (1996), no. 6, 995-1002.

[28] Stoker, J.J., Water waves, Interscience, New York, 1957.

[29] Vázquez, J.L., The porous medium equation. Mathematical theory, Oxford Mathematical Monographs, The Clarendon Press, Oxford University Press, Oxford, 2007.

[30] Vázquez, J.L., Zuazua, E., Large time behavior for a simplified $1 D$ model of fluid-solid interaction, Comm. Partial Differential Equations 28 (2003), no. 9-10, 1705-1738.

[31] Wrobel, L.C., Brebbia, C.A. (Eds.), Computational modelling of free and moving boundary problems, Vol. 1, Fluid flow, Proceedings of the First International Conference held 2-4 July, 1991, Southampton, U.K., Computational Mechanics Publications, Southampton, 1991.

E. Fernández-Cara: Dpto. E.D.A.N., Universidad de Sevilla, Aptdo. 1160, 41080 Sevilla, Spain

E-mail address: cara@us.es

Freddy Hernández: Inst. Matemática - Universidade Federal Fluminense, RJ, Brasil

E-mail address: freddyhernandez@id.uff.br 
Juan Límaco: Inst. Matemática - Universidade Federal Fluminense, RJ, Brasil E-mail address: jlimaco@vm.uff.br 